\title{
Effect of absent end diastolic flow velocity in the fetal umbilical artery on subsequent outcome
}

\author{
Pauline N A Adiotomre, Frank D Johnstone, Ian A Laing
}

\begin{abstract}
Sixty babies, delivered over a six and a half year period, who had absent end diastolic frequency (AEDF) in the umbilical artery, were studied. Individually matched control pregnancies for gestational age, birthweight, maternal clinical condition and date of delivery, in whom umbilical artery recordings showed end diastolic frequency, were also studied.

Matching was achieved in 36 cases. Neonates from case pregnancies showed no increase in necrotising enterocolitis, intraventricular haemorrhage, pneumothorax, neonatal death or bronchopulmonary dysplasia. However, they were significantly less likely to require ventilation for respiratory distress syndrome $(P=0.02)$.

Although AEDF indicates a fetus under vascular stress, this finding alone will include a spectrum of response in the baby, from the well compensated to the irreversibly damaged. Delivery at different points in the deteriorating fetal environment may explain discrepant study results. This intrauterine stress, by increasing fetal corticosteroid and thyroid hormones, may account for enhanced lung maturity. Predictions of neonatal course need to be based on more comprehensive awareness of fetal status.

(Arch Dis Child 1997;76:F35-F38)
\end{abstract}

Keywords: high risk pregnancies; end diastolic flow velocity; ventilation; respiratory distress syndrome.

Neonatal complications, particularly necrotising enterocolitis, are reportedly more likely where prenatal umbilical artery Doppler recordings show absent or reversed end diastolic frequencies (AREDF). However, these babies are usually delivered very premature from complicated pregnancies, and existing data do not exclude confounding problems.

The investigation of fetal circulation by Doppler ultrasound, first introduced by Fitzgerald and Drumm, ${ }^{1}$ is increasingly being used in high risk pregnancies. ${ }^{2}$ Abnormal fetal circulation shown by absent or reversed end diastolic flow velocity in the fetal umbilical artery or aorta suggests intrauterine fetal compromise. ${ }^{25}$ Absent end diastolic flow velocity is thought to result from increased downstream vascular resistance ${ }^{67}$ Circulatory redistribution is associated with absent end diastolic flow velocity, shown by decreased blood flow to the lung, intestines, kidneys, skin, and muscle, with blood diverted to the brain, myocardium and adrenals. ${ }^{3}$ This reduction in visceral perfusion has been associated with an increased risk of necrotising enterocolitis, cerebral haemorrhage, and neonatal morbidity. ${ }^{38}$

We studied retrospectively the clinical history and neonatal and subsequent outcome (in terms of bronchopulmonary dysplasia and mortality) of absent end diastolic flow velocity in a group of women with high risk pregnancies.

\section{Methods}

The case notes of 60 women and their babies were reviewed. They were selected over a six and a half year period (November 1985 to April 1992) as high risk pregnancies associated with absent end diastolic flow velocity from a record of Doppler studies. Controls consisted of women whose fetuses showed the presence of end diastolic flow velocity. Cases and controls were matched for gestational age (within one week), birthweight (within $250 \mathrm{~g}$ ), maternal clinical condition and date of delivery (within 18 months) from the Mentor computer database of antenatal and perinatal cases. Of the 898 potential control matches, only 36 controls fully met all the above criteria; the controls with the closest matches were chosen. Twenty four cases could not be matched because of non-availability of Doppler ultrasound results $(n=5)$, differences in maternal clinical conditions $(n=12)$, birthweight not within $250 \mathrm{~g}(\mathrm{n}=3)$, date of birth not within 18 months $(n=2)$ and non-availability of case notes $(n=2)$. Stillbirths were excluded from the neonatal outcome (one case and one control).

It was the policy of the obstetric unit to monitor high risk pregnancies for fetal compromise, using clinical assessment, ultrasound scan assessment of fetal growth, and biophysical profile and cardiotocographic tracings. The maternal histories were reviewed, taking note of maternal age, parity, previous obstetric and medical histories, complications of present pregnancy, maternal smoking and ingestion of alcohol and drugs taken during pregnancy. The reasons for delivery and evidence of fetal compromise were also noted. There was a rigorous protocol defining diagnosis of absent end diastolic frequencies. The high pass filter was set at $60 \mathrm{~Hz}$ to minimise artefactual absence of velocities due to concealment under the filter. The umbilical cord was visualised with ultrasound; waveforms were accepted only where a constant venous signal was obtained and the flow velocity waveform was sampled from three points in the abdomen to minimise the risk of abnormally high angles of incidence. In most cases the recording was repeated by another 
Table 1 Maternal characteristics, complications of pregnancy, caesarean section, and main maternal medication

\begin{tabular}{|c|c|c|c|c|}
\hline \multirow[b]{2}{*}{ Maternal charactericstics (No/\%): } & \multicolumn{2}{|l|}{ Cases } & \multicolumn{2}{|c|}{ Controls } \\
\hline & & & & \\
\hline Number & 36 & & 36 & \\
\hline Mean maternal age (years) (SD) & 27.0 & $(4.9)$ & 25.3 & (5.4) \\
\hline Nulliparous & 16 & $(44.4)$ & 20 & $(55.5)$ \\
\hline Smokers & 15 & $(44.1)(2)^{\star}$ & 11 & $(55.5)(2)^{\star}$ \\
\hline Mean No of cigarettes smoked (SD) & 11.33 & $(6.3)$ & 13.09 & $(3.1)$ \\
\hline Alcohol (>14 units per week) & 1 & $(2.8)$ & 0 & \\
\hline \multicolumn{5}{|l|}{ Pregnancy complications (No/\%): } \\
\hline No maternal disease with IUGR & 7 & $(19.4)$ & 8 & $(22.2)$ \\
\hline Hypertension in pregnancy & 26 & $(72.2)$ & 27 & $(75.0)$ \\
\hline Diabetes mellitus ${ }^{\dagger}$ & 3 & $(8.3)$ & 2 & $(5.5)$ \\
\hline Others ‡with IUGR & 2 & $(5.5)$ & 1 & $(2.8)$ \\
\hline \multicolumn{5}{|l|}{ Reasons for caesarean section (No/\%): } \\
\hline Fetal distress & 14 & $(38.8)$ & 7 & $(19.4)$ \\
\hline Maternal reasons + fetal distress & 5 & $(13.8)$ & 6 & $(16.6)$ \\
\hline Maternal reasons only & 7 & $(19.4)$ & 14 & $(38.8)$ \\
\hline Other fetal reasons & 4 & $(11.1)$ & 5 & $(13.8)$ \\
\hline \multicolumn{5}{|l|}{ Main maternal medications (No l\%): } \\
\hline Antihypertensives & 19 & $(52.78)$ & 22 & $(61.11)$ \\
\hline Dexamethasone & 4 & (11.11) & 3 & $(8.33)$ \\
\hline Insulin & 3 & $(8.33)$ & 2 & $(5.56)$ \\
\hline Others & 6 & $(16.67)$ & 8 & $(22.22)$ \\
\hline
\end{tabular}

* Information not available.

Others $\ddagger$ Maternal anti D and C antibody PROM x 3/52+ amnionitis + flu like illness Anti PLA antibody

PROM Prolonged rupture of membranes for more than 24 hours.

† Two cases and two controls with diabetes mellitus also had hypertension in pregnancy.

clinician within 24 hours and only where this confirmed AEDF was the diagnosis accepted. The mean resistance index of at least five technically acceptable waveforms had been recorded in the case notes of these mothers.

The neonatal histories were reviewed and the following noted: birthweight, sex, Apgar scores at 1 and 5 minutes, arterial or capillary blood gases at the age of 1 hour, where available, and blood pressure. The lowest platelet count and highest haematocrit within the first seven days of life were recorded. Clinical evidence of patent ductus arteriosus (PDA) and clinical with radiological evidence of respiratory distress syndrome (RDS) and necrotising enterocolitis were documented. Also documented were ultrasound scan evidence of intraventricular haemorrhage, neonatal and subsequent mortality up to the age of 2 years, and bronchopulmonary dysplasia. Bronchopulmonary dysplasia was defined as oxygen requirement at 28 days of life. Necrotising enterocolitis was diagnosed when there was abdominal distension with bile stained gastric aspirate or vomit in a sick baby who may have bloody mucousy stools, thrombocytopenia, and coagu-

Table 2

\begin{tabular}{lll}
\hline Neonatal characteristics & Cases & Controls \\
\hline Number & 36 & 36 \\
Median gestational age (weeks) (interquartile range) & $32.5(30.5-35)$ & $32.0(30-35)$ \\
Range of gestational age at delivery (weeks) & $27-38$ & $26-37$ \\
Median birthweight (g) (interquartile range) & $1367(1076-1798)$ & $1407(917-1849)$ \\
Birth weight $<10^{\text {th }}$ centile No (\%) & $30(83.3)$ & $26(72.2)$ \\
Sex (M:F) & $1: 0.8$ & $1: 1.77$ \\
Apgar scores $<5$ at $^{\ddagger}$ & $10(28.6)$ & $11(31.4)$ \\
1 minute No (\%) & $1(2.9)$ & $1(2.9)$ \\
5 minutes No (\%) & $7.33(12)^{\star}$ & $7.34(13)^{\star}$ \\
Mean pH at age 1 hour & & $3.1(16)^{\star}$ \\
Mean base deficit at age 1 hour $\ddagger$ & $2.3(12)^{\star}$ & $26(89.6)(6)^{\star}$ \\
Babies with first enteral feed & $24(82.7)(6)^{\star}$ & $3(10.3)(6)^{\star}$ \\
Within first day of life $(\%)^{\ddagger}$ & $4(13.8)(6)^{\star}$ & $3(10.3)(6)^{\star}$ \\
Feed intolerance (\%) $\ddagger$ & $4(13.5)(5)^{\star}$ & $8(27.6)(6)^{\star}$ \\
TPN administration $(\%)^{\ddagger}$ & $8(27.6)(6)^{\star}$ & \\
Babies who had EBM $(\%)^{\ddagger}$ & & \\
\hline
\end{tabular}

^ Information not available; EBM expressed breast milk; $\ddagger$ Stillbirths were excluded. lopathy with radiological findings of reduced bowel gas shadowing, thickening of bowel wall, and pneumatosis coli.

Statistical analysis was carried out using McNemar's test and the standard error of difference between means.

\section{Results}

The maternal characteristics, complications of pregnancy, and maternal medications were not statistically different in the two groups (table 1). Twelve cases and 11 controls experienced fetal distress in the absence of labour. Nine cases, however, had fetal distress during labour compared with two controls. Two of these cases required forceps delivery and the remaining seven were delivered by emergency caesarean section $(P=0.046)$.

The neonatal characteristics were also not significantly different (table 2). Eleven cases, compared with 19 controls, had respiratory distress syndrome. This difference was, however, not significant. Seventeen controls, compared with nine cases, required ventilation with or without paralysis or surfactant, which was significant $(P=0.02)$. Only four babies received replacement surfactant (one case and three controls).

The mean duration of ventilation was shorter in the case group (10.5 days), compared with that in the control group (21.3 days). Mean duration in oxygen, in both groups, was similar. Pneumothorax and pulmonary haemorrhage were rare complications in both groups. Thrombocytopenia occurred in about $30 \%$ of both study groups. Intraventricular haemorrhage, patent ductus arteriosus, and neonatal death were rare (table 3 ).

The feeding pattern was similar in both groups, and babies who required paralysis in the first few days of life received total parenteral nutrition (TPN) as feeds were omitted. One case and one control who had necrotising enterocolitis also received TPN. There was no increased association between necrotising enterocolitis and absent end diastolic flow velocity. The mean hospital stay was similar in both groups. Seven of the babies in the control group, and six of the cases, were transferred back to their referring hospitals. There was no difference in the rate of bronchopulmonary dysplasia (BPD) in both study groups. However, in the control group, the BPD was diagnosed at post mortem examination in two babies who had oxygen for less than 28 days (died before the age of 28 days). Seven of the controls and three of the babies with absent end diastolic flow velocity had congenital abnormalities.

On reviewing the 24 babies with absent end diastolic flow who had no controls, two were stillbirths. Of the remaining 22 babies, six died in the neonatal period and one sustained a cot death at the age of 3 months. A post mortem examination of this infant showed absent glycogen stores in the liver and reduced stores in the muscle. Only one baby had possible necrotising enterocolitis (suspected case). Table 4 shows the pregnancy complications, maternal medications, some neonatal charac- 
Table 3

\begin{tabular}{|c|c|c|c|c|c|}
\hline \multirow{2}{*}{$\begin{array}{l}\text { Neonatal outcome No (\%) } \\
\text { Number (\%) }\end{array}$} & \multicolumn{2}{|c|}{ Cases } & \multicolumn{2}{|c|}{ Control } & \multirow[t]{2}{*}{ P value } \\
\hline & 35 & & 35 & & \\
\hline RDS & 11 & $(31.4)$ & 19 & $(54.3)$ & 0.06 \\
\hline Ventilated & 9 & $(25.7)$ & 17 & $(48.6)$ & 0.02 \\
\hline Mean No of days ventilated & 10.6 & & 21.4 & & $>0.50$ \\
\hline Mean duration in $\mathrm{O}_{2}$ (days) & 28.4 & & 29.8 & & $>0.50$ \\
\hline Pneumothorax & 2 & $(5.7)$ & & $(2.9)$ & $>0.50$ \\
\hline Pulmonary haemorrhage & 0 & & & (8.6) & 0.13 \\
\hline DIC & 2 & $(5.7)$ & 1 & (2.9) & $>0.50$ \\
\hline Thrombocytopenia* & 8 & $(23.5)(1)^{\star}$ & 8 & $(25.0)(3)^{\star}$ & $>0.50$ \\
\hline $\mathrm{PCV} \geq 65 \%$ & 5 & $(14.7)(1)^{\star}$ & 1 & $(3.1)(3)^{\star}$ & 0.22 \\
\hline Hypotension $^{\ddagger}$ & 5 & $(16.1)(5)^{\star}$ & 6 & $(21.4)(7)^{\star}$ & $>0.50$ \\
\hline NEC & 1 & $(2.9)$ & 1 & $(2.9)$ & $>0.50$ \\
\hline IVH & 3 & $(8.6)$ & 5 & $(14.3)$ & $>0.50$ \\
\hline PDA & 5 & $(14.3)$ & 6 & $(17.1)$ & $>0.50$ \\
\hline $\mathrm{BPD}$ & 5 & $(14.3)$ & $5^{\dagger}$ & $(14.3)$ & $>0.50$ \\
\hline Neonatal death & 2 & $(5.7)$ & 3 & $(8.6)$ & $>0.50$ \\
\hline Death outside neonatal period & 0 & & 1 & & 0.31 \\
\hline Mean hospital stay in days & 34.5 & & 38.5 & & $>0.50$ \\
\hline
\end{tabular}

$\star$ Platelet count $<100 \times 10^{9} / 1^{\ddagger}{ }^{\ddagger}$ mean blood pressure $\mathrm{mmHg}<$ gestational age in weeks.

$\dagger$ Two controls confirmed at post mortem examination.

()$^{\star}$ Result not available.

PCV Packed cell volume; DIC Disseminated intravascular coagulopathy; BPD

Bronchopulmonary dysplasia; IVH intraventricular haemorrhage; PDA patent ductus arteriosus;

NEC necrotising enterocolitis

teristics, and neonatal outcome of these unmatched cases. These babies were more premature and of a lower birthweight when compared with those cases with controls. This would explain their apparent high morbidity and mortality.

\section{Discussion}

Absent end diastolic flow velocity in the fetal umbilical artery or aorta has been associated with high mortality, ${ }^{38}$ increased risk of necrotising enterocolitis, ${ }^{389}$ and haemorrhage. ${ }^{3}$ Our results, however, disagree with these findings. The main difference between our patients and controls was the presence or absence of forward end diastolic flow velocity, as this study was matched more rigorously than the others. There was no increased association with necrotising enterocolitis, intraventricular haemorrhage, pulmonary haemorrhage or neonatal death. In the group of 24 babies with AEDF who had no controls the morbidity and

Table 4 Summary of cases with no controls (No/\%)

\section{Pregnancy complication:}

No maternal disease with IUGR

Hypertension in pregnancy

Others with IUGR

Maternal medications:

Antihypertensives

Dexamethasone

Others

Some characteristics and outcome:

Median gestational age (interquartile range) (weeks)

Median birthweight (interquartile range) (g)

Birthweight $<10^{\text {th }}$ centile

Respiratory distress syndrome ${ }^{\star}$

Ventilated ${ }^{\star}$

IVH $^{\star}$

$\mathrm{PDA}^{\star}$

$\mathrm{NEC}^{\star}$

Pneumothorax ${ }^{\star}$

Pulmonary haemorrhage ${ }^{\star}$

PCV $\geq 65 \%$ *

Thrombocytopenia ${ }^{\star}$

Hypotension *

DIC ${ }^{\star}$

Bronchopulmonary dysplasia ${ }^{\star}$

Neonatal death ${ }^{\star}$

Stillbirths mortality appeared high as these babies were more premature and had lower birthweights. Our results may have been different if these babies had been included in the analyses. On the other hand, the effects of prematurity and lower birthweights could have been corrected for by rigorous matching of controls if they had been available. The cause of necrotising enterocolitis is multifactorial and its frequency varies between centres, ${ }^{10}$ and in addition, AEDF indicates a fetus under vascular stress, and delivery at different points in the deteriorating fetal environment may explain the discrepant study results.

In the study by McDonnell et al, ${ }^{11}$ seven of the 61 babies with absent or reversed end diastolic flow velocities (AREDFV) had necrotising enterocolitis compared with only one of the controls, although the difference was not statistically different. In keeping with our findings, they did not show an association with increased neonatal morbidity and mortality.

In our study there was a lower incidence of RDS among the babies with absent end diastolic flow velocity, but the difference was not significant. However, fewer of these babies required ventilation than in the control group and this difference was significant.

Fetuses with acute or chronic intrauterine hypoxia may mount up a mechanism which results in absent end diastolic flow velocity. The persistence of this insult might reverse the end diastolic flow. When this compensatory mechanism is overwhelmed, the fetus could present with fetal compromise, as shown by poor biophysical profile and/or abnormal cardiotocography. If the fetus is not delivered then, there could be an intrauterine death.

Intrauterine stress, as in postnatal life, should increase fetal catecholamines, corticosteroids, and thyroid hormones, which could enhance lung maturity. O'Brien has suggested that catecholamines, corticosteroids, thyroid hormones and perhaps prolactin influence the transition from non-functional to functional lung tissue. ${ }^{9}$ In recent years corticosteroids have been administered antenatally to mothers in pre-term labour to enhance fetal lung maturity by promoting surfactant synthesis and altering lung parenchymal structure. ${ }^{9}$ This is achieved by increasing lung elastin content and by decreasing alveolo-capillary permeability to serum proteins. Corticosteroids prevent RDS in $90 \%$ of pre-term babies delivered to women so treated. ${ }^{9}$

Some studies have suggested that thyrotrophin releasing hormones (TRH) administered antenatally potentiate the effects of corticosteroids in reducing the incidence of RDS. ${ }^{12}{ }^{13}$ The fetal pituitary is said to be able to respond to the maternal administration of TRH. ${ }^{14}$ In the small for gestational age fetuses the concentrations of thyroid stimulating hormone are said to be higher and those of total thyroxine and free thyroxine lower than in those appropriate for gestational age. ${ }^{14}$ It has also been suggested that the degrees of increase in TSH and the fall in thyroid hormones are related to the degree of fetal hypoxaemia and acidaemia, and that the low concentrations of 
thyroid hormones may have some beneficial effects by reducing oxygen requirement. ${ }^{14}$

Despite these, the well compensated fetus would probably still be able to increase its thyroid hormone values in response to stress which, in the long run, enhances fetal lung maturity and reduces the severity of RDS. In our study of high risk pregnancies the fetuses with absent end diastolic flow velocity might have had such a degree of intrauterine stress as to mount adequate cathecholamine and hormonal response (corticosteroid and thyroid hormone) to induce better lung maturity compared with those in the group with forward flow who have not had such a response.

Our thanks go to the medical records staff at the Simpson Memorial Maternity Pavilion for their assistance in obtaining the case notes and to Ms Margaret Reid for providing the computer printout of the controls matched with the cases. We are grateful to Miss Natalie Farrar for typing the script.

1 Fitzgerald DE, Drumm JE. Non-invasive measurement of human fetal circulation using ultrasound: a new method. BMF 1977; ii: $1450-1$.

2 Johnstone FD, Haddad NG, Hoskins P, McDicken W, Chambers S, Muir B. Umbilical artery Doppler flow velocity waveform: the outcome of pregnancies with absent end diastolic flow. Eur f Obstet Gynecol Reprod Biol 1988; 28:171-8.

3 Hackett GA, Campbell S, Gamsu H, Cohen Overbeek T, Pearce JMF. Doppler studies in the growth retarded fetus and prediction of neonatal necrotising enterocolitis, haem-
orrhage and neonatal morbidity. BMF 1987; 294:13-16.
4 Haddad NG, Johnstone FD, Hoskins PR, Chambers SE, Muir BB, McDicken WN. Umbilical artery Doppler waveform in pregnancies with uncomplicated intrauterine growth retardation. Gynecol Obstet Invest 1988;26:206-10.

5 Johnstone FD, Steel JM, Haddad NG, Hoskins PR, Greer IA, Chambers S. Doppler umbilical artery flow velocity waveform in diabetic pregnancy. $\mathrm{Br} \mathcal{F}$ Obstet Gynecol 1992;99:135-40.

6 Trudinger BJ, Giles W B, Cook CM, Bombar J, Collins J. Fetal umbilical artery velocity waveforms and placental
resistance; Clinical significance. $B r \quad \mathcal{F}$ Obstet Gynaecol 1985:92;23-30.

7 Skidmore R, Woodcock JP, Wells PNT. Physiological Interpretation of Doppler shift waveforms III. Ultrasound Interpretation of Doppler

8 Malcom G, Ellwood D, Devonald K, Beilby R, HendersonSmart D. Absent or reversed end diastolic flow velocity in the umbilical artery and necrotising enterocolitis. Arch Dis Child 1991;66:805-7.

9 McDonnell M, Serra-Serra V, Gaffney G, Redman CWG, Hope PI. Neonatal outcome after pregnancy complicated by abnormal velocity wave forms in the umbilical artery. Arch Dis Child 1994;70:F84-9.

10 Cosmi EU. Prenatal prevention of respiratory distress syndrome: new pharmacologic approaches. Early Hum Dev 1992; 29:283-6.

11 Kosloske A. Pathogenesis and prevention of necrotising enterocolitis: A hypothesis based on personal observation enterocolitis: A hypothesis based on personal observation

12 McDonnell M, Serra-Serra V, Gaffney G, Redman CWG, McDonnell M, Serra-Serra V, Gaffney G, Redman CWG,
Hope PI. Neonatal outcome after prengnancy complicated by abnormal velocity wave forms in the umbilical artery. Arch Dis Child 1994; 70: F84-9.

13 Knight DB, Liggins GC, Wealthall SR. A randomised, controlled trial of antepartum thyrotropin releasing hormone and betamethasone in the prevention of respiratory disease in pre-term infants. Am f Obstet Gynecol 1994;171:11-16.

14 de Zegher F, Spitz B, Devlieger H. Prenatal treatment with Thyrotrophin releasing hormone to prevent neonatal Thyrotrophin releasing hormone to prevent ne
respiratory distress. Arch Dis Child 1992;67:450-4.

15 Thorpe-Beeston JG, Nicolaides KH, McGregor AM. Fetal thyroid function. Thyroid 1992;2:207-17. 